Original

\title{
Group cognitive-behavioral intervention for patients with burning mouth syndrome
}

\author{
Osamu Komiyama ${ }^{1,2)}$, Hitoshi Nishimura1), Yasuhide Makiyama1), Takashi Iida ${ }^{1,2)}$, \\ Ryoko Obara1,2), Masamichi Shinoda'3), Masayuki Kobayashi4), Noboru Noma5), \\ Osamu Abe' ${ }^{6}$, Antoon De Laat7), and Misao Kawara ${ }^{1,2)}$ \\ 1)Orofacial and Head Pain Clinic, Nihon University School of Dentistry at Matsudo, Matsudo, Japan \\ 2)Department of Oral Function and Rehabilitation, Nihon University School of Dentistry at Matsudo, \\ Matsudo, Japan \\ 3)Department of Physiology, Nihon University School of Dentistry, Tokyo, Japan \\ 4)Department of Pharmacology, Nihon University School of Dentistry, Tokyo, Japan \\ 5)Department of Oral Diagnostic Sciences, Nihon University School of Dentistry, Tokyo, Japan \\ 6)Department of Radiology, Nihon University School of Medicine, Tokyo, Japan \\ 7)Department of Oral Health Sciences, KU Leuven and Dentistry, University Hospitals, Leuven, Belgium
}

(Received August 1, 1012; Accepted November 30, 2012)

\begin{abstract}
This study was conducted to assess the psychological characteristics of, and determine the effectiveness of group cognitive-behavioral (CB) treatment for, patients with burning mouth syndrome (BMS). The baseline characteristics of 24 female patients (age $69.7 \pm 5.9$ years) and an identical number of healthy female control subjects (age 69.2 \pm 5.5 years) were compared. The patient group had significantly higher anxiety scores $(P<0.05)$ at baseline. A brief group CB intervention was delivered in a small-group format. Two sessions were planned 6 months apart. A numeric rating scale (NRS) was used to assess pain intensity. Anxiety was evaluated using a state and trait anxiety inventories. Present pain intensity decreased after both the first and second sessions. The session effect was significant $(P=0.02)$, but no repeat effect was found $(P=0.19)$. The state anxiety inventory score also decreased after the second session. The session effect was significant $(P<0.01)$, as was the repeat effect $(P<0.01)$. The trait anxiety inventory score decreased after the second session,
\end{abstract}

Correspondence to Dr. Osamu Komiyama, Department of Oral Function and Rehabilitation, Nihon University School of Dentistry at Matsudo, 2-870-1 Sakaecho-nishi, Matsudo, Chiba 271-8587, Japan

Fax:+81-47-360-9615 E-mail: komiyama.osamu@nihon-u.ac.jp and the session effect was significant $(P=0.013)$, but the repeat effect was not $(P=0.93)$. The results suggest that a brief group $C B$ intervention reduces pain intensity and anxiety in patients with BMS.

(J Oral Sci 55, 17-22, 2013)

Keywords: burning mouth syndrome; glossodynia; cognitive behavioral intervention; orofacial pain; anxiety.

\section{Introduction}

Burning mouth syndrome (BMS) is a chronic, intractable pain condition characterized by a burning sensation or other dysesthesia of the oral mucosa, without accompanying abnormal clinical or laboratory findings (1). The International Association for the Study of Pain (IASP) has identified BMS as a "distinctive nosological entity" characterized by "unremitting oral burning or similar pain in the absence of detectable oral mucosa changes"

(2). The tongue is the primary location of the burning complaint in most patients, and thus, this condition has also been called glossodynia (3).

Conclusive clinical trials are sparse (4), and therefore, the condition has no established treatment protocol (4). Three well-designed and controlled studies of various classes of antidepressants $(5,6)$ and a nonsteroidal anti- 
inflammatory oral rinse (7) yielded negative results. Many other treatments, such as sialogogues, topical anesthesia, oral rinses, antidepressants, benzodiazepines, anticonvulsives, anxiolytics, and psychological therapy have been proposed based on the results of open studies or on clinical experience (8-12). Gremeau-Richard et al. (13) showed that topical clonazepam improved stomatodynia symptoms, but this treatment was not effective for all participants, for reasons that are poorly understood. The lack of any significant improvement and the side effects associated with many of these treatments make the management of BMS controversial.

Cognitive-behavioral (CB) therapy aims to decrease the maladaptive cognitions and behaviors of patients and increase adaptive cognitions and behaviors. Such treatments are effective for a variety of chronic pain problems (14-16), and Bergdahl et al. (8) reported that, as compared with a control group, the intensity of BMS-related pain decreased after CB therapy.

$\mathrm{CB}$ therapy has proven effective for pain reduction in BMS patients (17). In a nonrandomized open-label study, Pekinar et al. (18) reported that moclobemide was effective in treating BMS and showed that BMS patients had significantly higher anxiety and depression scores than did controls. However, no report has described the psychological effect of group CB intervention for BMS patients. We therefore investigated the effectiveness of group $\mathrm{CB}$ treatment for improving pain and anxiety in BMS patients.

\section{Methods}

\section{Subjects}

The study subjects were recruited from patients who sought treatment in our clinic for burning sensation of the tongue from 2007 through 2009. On the basis of the IASP criteria for BMS (2), the inclusion criteria were presence of an isolated complaint of chronic pain in the tongue or oral mucosa and a normal clinical examination. Pain was present for more than 3 months in all patients, was continuous throughout all or part of the day, without paroxysms, and did not follow a nerve trajectory. Patients presenting with an organic condition that could be considered a potential causative factor, such as diabetes or anemia, were excluded, and patients who had received treatment by a psychiatrist were also excluded. For all subjects, local or systemic conditions were evaluated using laboratory examinations (eg, blood cell count, serum iron foliate level, detection of Candida species). Because BMS is more prevalent among women, 24 female subjects (age 69.7 \pm 5.9 years) were included. All subjects had symptoms restricted to the tongue only. The average duration of pain was $38.3 \pm 14.4$ months, and the average duration of previous treatment was $16.4 \pm 10.7$ months. Because patients reported some benefit from previous treatment, that treatment (ie, mouth rinse and drug administration) was continued during the present study. The medications used were an antiepileptic (12 subjects), antidepressants (five subjects), and anxiolytics (three subjects). The drugs were used either alone or in combination, and the medication regimen was not changed during the study period.

To compare the baseline psychological characteristics of subjects, the age- and sex-matched normal control subjects (NC; 24 women, age $69.2 \pm 5.5$ years) were recruited from patients attending a periodontal re-call program. All NC subjects were without chronic pain or psychiatric problems.

All subjects were informed about the study in a standardized way. The institutional ethics committee approved the study (EC07-003), and the study has adhered to the guidelines set out by the Declaration of Helsinki.

\section{Brief group CB intervention}

The $\mathrm{CB}$ intervention was delivered in a small-group format. Both a dentist and a neurologist gave a 20 -min presentation (total: $40 \mathrm{~min}$ ). They used a set of materials to provide information on the nature and typical course of BMS, the biological and behavioral management of BMS, the relationship between the burning sensation and psychophysiological aspects of stress, the basics of pain physiology (with an emphasis on chronic pain), and an introduction to cognitive and behavioral pain and stress-coping strategies. All patients learned, and had an opportunity to briefly practice, a progressive relaxation method. After receiving an explanation of this information, patients took part in an approximately 1-hr group discussion of their experiences, eg, symptoms, onset of symptoms, and their pain and distress. A second CB intervention session was planned for 6 months later, and the same interventions were performed.

\section{Measurements}

The numeric rating scale (NRS) was used to assess present pain intensity, worst pain intensity in the past 6 months, and disturbance to daily life in the past 6 months. Subjects received precise instruction on how to rate pain intensity on a 10-point numeric rating scale (NRS) from 0 (no pain) to 10 (greatest pain imaginable) for pain intensity and from 0 (no disturbance) to 10 (greatest disturbance imaginable) for disturbance to daily life with regard to eating (such as mastication, swallowing, 

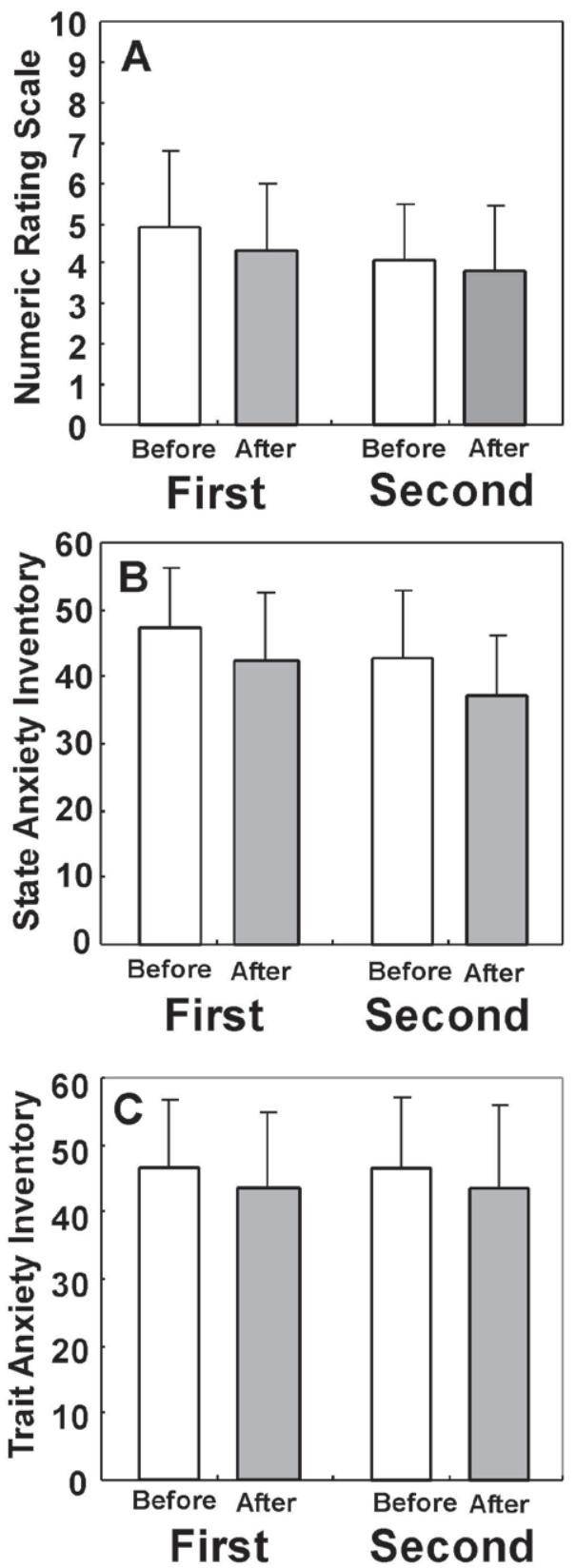

Fig. 1 Numeric rating scale of present pain intensity (A), state anxiety inventory score $(\mathrm{B})$, and trait anxiety inventory score $(C)$. There was a significant session effect $(P=0.023)$ on present pain intensity but no repeat effect $(P=0.198)$. There was a significant session effect $(P<0.001)$ on state anxiety inventory score and a repeat effect $(P=0.007)$. There was also a significant session effect $(P=0.013)$ on trait anxiety inventory score but no repeat effect $(P=0.930)$. Error bars indicate standard deviation.

etc.), sleep disturbance, and job performance. Present pain intensity, worst pain intensity in the past 6 months, and disturbance to daily life in the past 6 months were assessed before the CB session, and present pain intensity was assessed again after the session.
The Japanese version of the state-trait anxiety inventory (STAI) was used to evaluate anxiety before and after the session $(19,20)$.

\section{Statistical analysis}

Descriptive statistics were used to summarize all measurements. Because the response variables had a normal distribution (Shapiro-Wilkes test) and were assumed to be continuous, the mean and SD of the NRS value and STAI score were calculated. One-way ANOVA was used to compare the baseline characteristics of the control group and patient group in terms of worst pain intensity and disturbance to daily life in the past 6 months. Repeated two-way analysis of variance (ANOVA) was used to detect the session effect (difference between the values before and after a session) and repeat effect (changes between the first and second sessions: 6-month interval) on present pain intensity and STAI score. Bonferroni correction for multiple comparisons was applied, and the level of statistical significance was set at $P<0.05$. Means $\pm \mathrm{SD}$ are presented in the text and tables. All analyses were conducted using the SPSS software program (SPSS 12.0 for Windows, SPSS Inc., Chicago, IL, USA).

\section{Results}

\section{Pain intensity and disturbance to daily life}

The control group was pain-free in the orofacial region. Present pain intensity among the patient group decreased from $4.9 \pm 1.8$ to $4.3 \pm 1.7$ after the first session, and from $4.2 \pm 1.9$ to $3.9 \pm 1.9$ after the second session (Fig. 1). Two-way ANOVA showed a significant session effect $(P$ $=0.023)$, but the decrease between the first and second sessions (repeat effect) was not significant $(P=0.198)$.

The worst pain intensity in the past 6 months significantly decreased from $6.7 \pm 2.1$ to $5.4 \pm 2.3(P=0.042)$ from after the first session to after the second session (a 6-month interval), and disturbance to daily life in the past 6 months also significantly decreased, from $4.8 \pm 2.2$ to $3.6 \pm 1.7(P=0.037)$.

\section{State and trait anxiety}

The state anxiety inventory score of the patients $(47.3 \pm$ 9.0) was higher than that of the controls $(38.2 \pm 6.7 ; P=$ 0.001 ); however, the trait anxiety inventory scores of the control and patient groups were comparable: $47.7 \pm 11.2$ among controls vs $46.7 \pm 9.0$ among patients.

Among patients, the state anxiety inventory score decreased from $47.3 \pm 9.0$ to $41.6 \pm 10.2$ after the first session, and from $43.6 \pm 11.3$ to $37.8 \pm 11.3$ after the second session. The session effect was significant $(P<$ $0.001)$, as was the repeat effect $(P=0.007)$. The trait 
anxiety inventory score in the patient group decreased from $46.7 \pm 9.0$ to $43.5 \pm 11.1$ after the first session, and from $46.4 \pm 10.4$ to $43.6 \pm 12.1$ after the second session. There was a significant session effect $(P=0.013)$, but no repeat effect $(P=0.930)$.

\section{Narrative impressions after $C B$ session}

Narrative impressions after the $\mathrm{CB}$ intervention were written on the questionnaire. Representative responses included, "My anxiety was reduced, since I understand that this illness and pain are common and do not affect only me," and "I learned to live better with this pain, as I saw that many people are in the same situation." Also, there were many comments regarding the patient's newfound peace of mind and sympathy for other patients after the $\mathrm{CB}$ intervention.

\section{Discussion}

A brief group CB intervention was designed to increase knowledge of the causes and treatment of BMS, to provide skills in self-monitoring of the condition, and to introduce behavioral strategies to manage chronic, persistent BMS pain. Pain intensity and disturbance to daily life significantly decreased from the first to the second $\mathrm{CB}$ session. The state anxiety inventory score also decreased after the $\mathrm{CB}$ session and approached the control value.

The pathophysiology of BMS is largely unexplained, and this is directly related to the difficulty experienced in managing patients with BMS. Several reports suggest that BMS is likely to be a neuropathic pain condition $(21,22)$. Some authors have suggested that peripheral neuropathic pathophysiological mechanisms have a role (23-27), while others believe that central neuropathic mechanisms are primarily involved (28-31). Bergdahl et al. (8) reported that cognitive therapy for BMS patients resulted in lower pain intensity as compared with an untreated control group. They concluded that resistant BMS is probably of psychological origin in some patients. Our results also showed a good response to brief group $\mathrm{CB}$ intervention in patients with persistent BMS.

The anxiety score was higher in patients than in the controls, thus confirming previous findings, which indicated that psychological factors are often associated with BMS (32-34). Nevertheless, the causal relationship between anxiety and BMS is unclear. Exposure to prolonged stress, such as that caused by a chronic burning sensation, might affect psychological functioning (35). However, further investigation is needed to answer this question. State and trait anxiety scores were lower after the CBT intervention and approached control values, and pain intensity also decreased. Therefore, the beneficial effects of group $\mathrm{CB}$ intervention may be mediated by psychological factors, in combination with pain perception.

A randomized controlled trial found that psychotherapy or cognitive therapy sessions provided for 1 hour per week over 12 to 15 weeks were beneficial in reducing BMS pain intensity for up to 6 months (8). Another study showed some improvement from psychotherapy over 2 months, and a significant improvement when this treatment was combined with $\alpha$-lipoic acid therapy (36). However, the authors of that study used only a visual analogue scale to evaluate pain and burning sensation.

Pekiner et al. (18) assessed the effectiveness of the antidepressant moclobemide on burning pain and the psychological status of BMS patients. State anxiety inventory score significantly decreased from $40.2 \pm$ 9.9 to $36.4 \pm 8.0$, and trait anxiety inventory score also significantly decreased, from $46.7 \pm 8.9$ to $41.7 \pm 18.6$, after start of treatment. In our study, state anxiety score significantly decreased from $47.3 \pm 9.0$ to $37.8 \pm 11.3$, and trait anxiety inventory score significantly decreased from $46.7 \pm 9.0$ to $43.6 \pm 12.1$. These values approached those in the control group and were accompanied by a reduction in pain intensity. Thus, $\mathrm{CB}$ intervention may be effective for improving state anxiety inventory score. If so, a brief group cognitive-behavioral intervention can be part of effective management of chronic BMS patients and is a useful method for reducing anxiety in such patients.

Individual and group cognitive therapy have been shown to be equally effective in managing chronic pain (37): there was no differencs in treatment effect between individual therapy and group CB sessions. However, as seen in the narrative impressions of our patients, group $\mathrm{CB}$ intervention may be more helpful in managing persistent pain in BMS patients, because it provides mutual psychological support. Detailed studies of the effect duration of CB therapies on complaints of burning sensation are needed.

The results of this study suggest that a brief group cognitive-behavioral intervention was effective in decreasing pain and anxiety in BMS patients. Reduction of anxiety may also be helpful in reducing pain intensity in BMS patients.

\section{Acknowledgments}

This study was supported by a Grant-in-Aid for Scientific Research (C 20592285) from the Japanese Society for the Promotion of Science. 


\section{References}

1. Grushka M, Epstein JB, Gorsky M (2002) Burning mouth syndrome. Am Fam Physician 65, 615-620.

2. Merskey H, Bogduk N (1994) Classification of chronic pain: descriptions of chronic pain syndromes and definitions of pain terms. 2nd ed, IASP Press, Seattle, 209-214.

3. Bergdahl B, Bergdahl J (1999) Burning mouth syndrome: prevalence and associated factors. J Oral Pathol Med 28, 350-354.

4. Zakrzewska JM, Forssell H, Glenny AM (2003) Interventions for the treatment of burning mouth syndrome: a systematic review. J Orofac Pain 17, 293-300.

5. Loldrup D, Langemark M, Hansen HJ, Olesen J, Bech P (1989) Clomipramine and mianserin in chronic idiopathic pain syndrome. A placebo controlled study. Psychopharmacology 99, 1-7.

6. Tammiala-Salonen T, Forssell H (1999) Trazodone in burning mouth pain: a placebo-controlled, double-blind study. J Orofac Pain 13, 83-88.

7. Sardella A, Uglietti D, Demarosi F, Lodi G, Bez C, Carrassi A (1999) Benzydamine hydrochloride oral rinses in management of burning mouth syndrome. A clinical trial. Oral Surg Oral Med Oral Pathol Oral Radiol Endod 88, 683-686.

8. Gorsky M, Silverman S, Chinn H (1991) Clinical characteristics and management outcome in the burning mouth syndrome. An open study of 130 patients. Oral Surg Oral Pathol Med Oral Pathol 72, 192-195.

9. Bergdahl J, Anneroth G, Perris H (1995) Cognitive therapy in the treatment of patients with resistant burning mouth syndrome: a controlled study. J Oral Pathol Med 24, 213-215.

10. Huang W, Rothe MJ, Grant-Kels JM (1996) The burning mouth syndrome. J Am Acad Dermatol 34, 91-98.

11. Formaker BK, Mott AE, Frank ME (1998) The effects of topical anesthesia on oral burning in burning mouth syndrome. Ann N Y Acad Sci 855, 776-780.

12. Grushka M, Epstein J, Mott A (1998) An open-label, dose escalation pilot study of the effect of clonazepam in burning mouth syndrome. Oral Surg Oral Med Oral Pathol Oral Radiol Endod 86, 557-561.

13. Gremeau-Richard C, Woda A, Navez ML, Attal N, Bouhassira D, Gagnieu MC, Laluque JF, Picard P, Pionchon P, Tubert S (2004) Topical clonazepam in stomatodynia: a randomised placebo-controlled study. Pain 108, 51-57.

14. Keefe FJ, Caldwell DS (1997) Cognitive behavioral control of arthritis pain. Med Clin North Am 81, 277-290.

15. Morley S, Eccleston C, Williams A (1999) Systematic review and meta-analysis of randomized controlled trials of cognitive behaviour therapy and behaviour therapy for chronic pain in adults, excluding headache. Pain 80, 1-13.

16. Eccleston C, Morley S, Williams A, Yorke L, Mastroyannopoulou K (2002) Systematic review of randomised controlled trials of psychological therapy for chronic pain in children and adolescents, with a subset meta-analysis of pain relief. Pain 99, 157-165.

17. Patton LL, Siegel MA, Benoliel R, De Laat A (2007) Manage- ment of burning mouth syndrome: systematic review and management recommendations. Oral Surg Oral Med Oral Pathol Oral Radiol Endod 103, Suppl, S39.e1-13.

18. Pekiner FN, Gumru B, Ozbayrak S (2008) Efficacy of moclobemide in burning mouth syndrome: a nonrandomized, open-label study. J Orofac Pain 22, 146-152.

19. Gorsuch RL, Lushene R, Spielberger CD, Vagg PR (1983) Manual for the State-Trait Anxiety Inventory (Form Y). Consulting Psychologists Press, Palo Alto.

20. Nakazato K, Shimonaka Y (1989) The Japanese State-Trait Anxiety Inventory: age and sex differences. Percept Mot Skills 69, 611-617.

21. Lauria G, Majorana A, Borgna M, Lombardi R, Penza P, Padovani A, Sapelli P (2005) Trigeminal small-fiber sensory neuropathy causes burning mouth syndrome. Pain 115, 332-337.

22. Yilmaz Z, RentonT, Yiangou Y, Zakrzewska J, Chessell IP, Bountra C, Anand P (2007) Burning mouth syndrome as a trigeminal small fibre neuropathy: increased heat and capsaicin receptor TRPV1 in nerve fibres correlates with pain score. J Clin Neurosci 14, 864-871.

23. Lauritano D, Spadari F, Formaglio F, Zambellini Artini M, Salvato A (1998) Etiopathogenic, clinical-diagnostic and therapeutic aspects of the burning mouth syndrome. Research and treatment protocols in a patient group. Minerva Stomatol 47, 239-251.

24. Heckmann SM, Heckmann JG, Hilz MJ, Popp M, Marthol H, Neundörfer B, Hummel T (2001) Oral mucosal blood flow in patients with burning mouth syndrome. Pain 90, 281-286.

25. Femiano F, Scully C (2002) Burning mouth syndrome (BMS): double blind controlled study of alpha-lipoic acid (thioctic acid) therapy. J Oral Pathol Med 31, 267-269.

26. Grushka M, Epstein JB, Gorsky M (2003) Burning mouth syndrome and other oral sensory disorders: a unifying hypothesis. Pain Res Manag 8, 133-135.

27. Nagler RM, Hershkovich O (2004) Sialochemical and gustatory analysis in patients with oral sensory complaints. J Pain 5, 56-63.

28. Jääskeläinen SK, Forssell H, Tenovuo O (1997) Abnormalities of the blink reflex in burning mouth syndrome. Pain 73, 455-460.

29. Forssell H, Jääskeläinen S, Tenovuo O, Hinkka S (2002) Sensory dysfunction in burning mouth syndrome. Pain 99, 41-47.

30. Hagelberg N, Forssell H, Aalto S, Rinne JO, Scheinin H, Taiminen T, Någren K, Eskola O, Jääskeläinen SK (2003) Altered dopamine D2 receptor binding in atypical facial pain. Pain 106, 43-48.

31. Hagelberg N, Forssell H, Rinne JO, Scheinin H, Taiminen T, Aalto S, Luutonen S, Någren K, Jääskeläinen S (2003) Striatal dopamine D1 and D2 receptors in burning mouth syndrome. Pain 101, 149-154.

32. Grushka M, Sessle BJ, Miller R (1987) Pain and personality profiles in burning mouth syndrome. Pain 28, 155-167.

33. Lamb AB, Lamey PJ, Reeve PE (1988) Burning mouth syndrome: psychological aspects. Br Dent J 165, 256-260. 
34. Lamey PJ, Lamb AB (1988) Prospective study of aetiological factors in burning mouth syndrome. Br Med J 296, 1243-1246.

35. Lamey PJ, Freeman R, Eddie SA, Pankhurst C, Rees T (2005) Vulnerability and presenting symptoms in burning mouth syndrome. Oral Surg Oral Pathol Oral Radiol Endod 99, 48-54.

36. Femiano F, Gombos F, Scully C (2004) Burning mouth syndrome: open trial of psychotherapy alone, medication with alpha-lipoic acid (thioctic acid), and combination therapy. Med Oral 9, 8-13.

37. Turner-Stokes L, Erkeller-Yuksel F, Miles A, Pincus T, Shipley M, Pearce S (2003) Outpatient cognitive behavioral pain management programs: a randomized comparison of a group-based multidisciplinary versus an individual therapy model. Arch Phys Med Rehabil 84, 781-788. 